\title{
Filigrane
}

Écoutes psychanalytiques

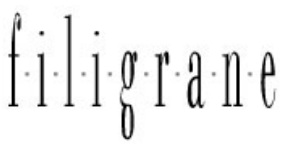

\section{Un drapeau taliban, at home}

\section{Anthony Bourgeault}

Volume 25, numéro 2, 2016

Le sujet de la violence

URI : https://id.erudit.org/iderudit/1039648ar

DOI : https://doi.org/10.7202/1039648ar

Aller au sommaire du numéro

\section{Éditeur(s)}

Revue Santé mentale au Québec

ISSN

1192-1412 (imprimé)

1911-4656 (numérique)

Découvrir la revue

\section{Citer cet article}

Bourgeault, A. (2016). Un drapeau taliban, at home. Filigrane, 25(2), 61-90. https://doi.org/10.7202/1039648ar

\section{Résumé de l'article}

Cherchant une oeuvre d'art pour combler un mur inoccupé de sa maison, l'auteur rencontre une série de drapeaux associés à des groupes armés. Cette rencontre imprévue devient le lieu propice à un enchaînement d'impressions de l'ordre de l'inquiétante étrangeté. À travers un parcours qui la mène de la Bosnie à une sexualité féroce, en passant par l'oeil automatique captant un terrible selfie, la violence du monde fait résonner une part d'inassimilable, aux limites de l'intimité. En même temps vient la tentation de se ressaisir partiellement, dans l'essai de comprendre ce qui arrive. L'auteur interroge alors l'origine des rêveries éveillées, y reconnaissant l'apport de l'autre. Il considère aussi le pulsionnel délié et la blessure identitaire, sous-jacents à plusieurs actes de violence. Les langues théorique et philosophique utilisées en ces circonstances demeurent néanmoins en conversation avec une expérience affective vivace, qui les relance constamment. Placée sous le signe du discours amoureux et de la préservation du sentiment, la présente écriture souhaite exprimer un mouvement de pensée, dans le courant duquel une langue intelligible trame avec des mots traumatiques. L'implantation d'une base vitale, d'un " drap-peau ", a-t-elle rendu l'élaboration possible, transcendant la force d'attraction qu'exerce la violence, sans en trahir toute l'intensité destructrice?
Ce document est protégé par la loi sur le droit d'auteur. L'utilisation des services d'Érudit (y compris la reproduction) est assujettie à sa politique d'utilisation que vous pouvez consulter en ligne.

https://apropos.erudit.org/fr/usagers/politique-dutilisation/ 


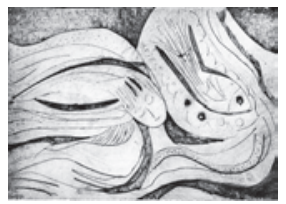

\title{
Un drapeau taliban, at home
}

\author{
Anthony Bourgeault
}

\begin{abstract}
RÉSUMÉ: Cherchant une œuvre d'art pour combler un mur inoccupé de sa maison, l'auteur rencontre une série de drapeaux associés à des groupes armés. Cette rencontre imprévue devient le lieu propice à un enchaînement d'impressions de l'ordre de l'inquiétante étrangeté. À travers un parcours qui la mène de la Bosnie à une sexualité féroce, en passant par l'œil automatique captant un terrible selfie, la violence du monde fait résonner une part d'inassimilable, aux limites de l'intimité. En même temps vient la tentation de se ressaisir partiellement, dans l'essai de comprendre ce qui arrive. L'auteur interroge alors l'origine des rêveries éveillées, y reconnaissant l'apport de l'autre. Il considère aussi le pulsionnel délié et la blessure identitaire, sous-jacents à plusieurs actes de violence. Les langues théorique et philosophique utilisées en ces circonstances demeurent néanmoins en conversation avec une expérience affective vivace, qui les relance constamment. Placée sous le signe du discours amoureux et de la préservation du sentiment, la présente écriture souhaite exprimer un mouvement de pensée, dans le courant duquel une langue intelligible trame avec des mots traumatiques. L'implantation d'une base vitale, d'un «drap-peau », a-t-elle rendu l'élaboration possible, transcendant la force d'attraction qu'exerce la violence, sans en trahir toute l'intensité destructrice?
\end{abstract}

Mots clés: Art, rêverie, sexualité, pulsionnalité, violence

Savoir que l'on n'écrit pas pour l'autre, savoir que ces choses que je vais écrire ne me feront jamais aimer de qui j'aime, savoir que l'écriture ne compense rien, ne sublime rien, qu'elle est précisément là où tu n'es pas, c'est le commencement de l'écriture.

Roland Barthes, Fragments d'un discours amoureux

e vis avec la violence. Violence du monde, monde humain, le mien. Récemment, j'ai rencontré une œuvre d'art qui en est empreinte. Elle s'intitule Home-made, Home-grown, de l'artiste visuel canadien Miles Collyer, et rassemble des drapeaux associés à des groupes armés. Dans l'aura de cette rencontre, d'autres réalités et une fiction s'invitèrent. D’abord, terrorisme international, puis, une guerre mondiale, la révolution américaine, la guerre de Bosnie, le «barebacking», sans compter le meurtre théâtral 
d'un amant. Autant de relais sur lesquels la présente élaboration s'est mise à rebondir. Oui, la violence mobilise. Dirait-on, pour reprendre l'expression de Pontalis (1990), «force d'attraction»?

Bien que la réflexion naissante puisse se trouver attirée en sa direction, la violence en tant que telle n'y sera pas systématiquement examinée. Ni la violence en général, ni une violence spécifique ne seront expliquées. J’essaie (sans y parvenir tout à fait) de ne pas écrire sur la violence. «Écrire sur quelque chose [par opposition à exprimer quelque chose], c'est le périmer» (Barthes, 1977, p. 114). Périmer, du latin «perimere»: abolir, détruire.

Dans le cas particulier de l'écriture, périmer la violence signifie-t-il en affaiblir la charge affective? La dégonfler, la dégrader? En même temps, dans le monde humain, le projet de périmer la violence, comme dans la guerre au terrorisme, de l'abolir, et ce faisant, d'éradiquer le sujet de la violence, œuvre-t-il efficacement à son éloignement? En réagissant au mal par une force annihilante, encore violente, un tel projet reporterait-il plutôt le mal? Cette problématique brûle sans contredit de complexité. Or, quel sujet s'agira-t-il ici d'explorer, et comment? Je dirais qu'il se trouve du côté d'une rencontre avec l'œuvre d'art. Mais encore?

Pour mieux l'appréhender, nous nous référerons au mémoire de Miles Collyer (2015). Il y traite du sujet de son exposition How do you surrender a drone? Et vraisemblablement, déjà au sein du travail entourant Home-made, Home-grown, le sujet tient une place similaire. Il affirme:

My subject cannot be named directly but every created thing in this exhibition in someway speaks about this subject, so it is possible to know something about it by way of analogy. Analogy in this sense is the likeness or resonance between my subject and what has been created here. (Collyer, 2015, p. 1)

L'artiste postule la complexité, l'intangibilité et l'imprécision du sujet de son œuvre. Inaccessible directement, le sujet retentit alors dans les images et les objets signifiants qui composent l'œuvre. Visuellement, ces images et objets résonnent aussi entre eux.

En outre, dans une perspective psychanalytique, Rolland (2006) étudie longuement la production d'analogies. Il la comprend comme un effet de l'inconscient. Exclu du système de la langue, l'inconscient se manifesterait de façon indirecte, au travers de jeux de correspondances qui signaleraient une autre réalité, sous-jacente à la réalité énoncée. Une autre réalité, sousjacente à la réalité représentée et à la réalité perçue, convient-il d'ajouter. 
Dès lors, le sujet intangible, voire présent absent, que Collyer évoque peut-il avoir rapport à l'inconscient?

Inspiré par la démarche de l'artiste, je tends à concevoir le sujet de mon écriture comme étant une présence, absente du visible ou du lisible, mais avec laquelle elle correspond sans doute. Plutôt que renforcer le savoir, la présente écriture souhaite exprimer un mouvement de la pensée, en préservant l'affect qui ne se laisse pas rationaliser. «Mais l'écriture et la lecture sont lentes qui s'avancent à reculons dans la direction de la chose inconnue à l'intérieur», formule Lyotard (1988, p. 14). Lire et écrire, tâtonner une énigme, vivre l'étranger plutôt que le détruire; on n'en ressort pas indemne.

Certes, la rencontre avec l'œuvre d'art aurait bien pu être vécue sans être enregistrée par l'écriture. Cela aurait pu se passer sans l'éclairage de lectures de référence, et sans réécritures incessantes. Mais s'agirait-il, en ce cas, de la même rencontre? Si «l'écriture», telle que la conçoit Lyotard, réalise «cet effort pour inscrire la trace d'une chose qui nous affecte et nous déborde» (Bernard, 2014, p. 11), une certaine mémoire n’advient pas sans elle.

Divers niveaux sont interpellés et mis au travail dans la démarche actuelle, entretenant la conversation entre l'intime et le théorique, entre le traumatique et l'intelligible. Cette démarche rassemble les pensées hétérogènes qui fréquentent mon esprit, pensées traduites en des phrases puisées à différents genres de discours. Ainsi, le récit anecdotique côtoie la spéculation psychanalytique. La rêverie voisine le commentaire de l'historien de l'art. Le mythe historique croise la méditation du philosophe. Et, parmi ces discours, au travers de la parole qui s'écrit, percent, à l'occasion, les extraits d'un discours amoureux.

Le discours amoureux est nommé ainsi, comme s'il constituait un discours à part. L'essai du sémiologue Roland Barthes (1977) m’en suggère l'idée. Le discours amoureux délimite-t-il son propre genre discursif? Je ne saurais trancher. Néanmoins, lisant la préface à ses Fragments, je comprends que le discours amoureux témoigne d'une structure distinctive. Il se définit par la place depuis laquelle son affirmation a lieu: «la place de quelqu'un qui parle en lui-même, amoureusement, face à l'autre (l'objet aimé), qui ne parle pas» (Barthes, 1977, p. 7). En quelque sorte, le discours amoureux, tel que décrit par le sémiologue, s'apparente à un discours intérieur. $\mathrm{Ou}$, en termes structuraux: le discours amoureux «donne à lire une place de parole» (p. 7), et cette place suppose l'épreuve d'une intériorité, l'existence d'un «en soi-même», et la possibilité du silence de l'autre. 
Le discours amoureux n'est pas cité comme un genre parmi d'autres. Reflète-t-il l'horizon, ou bien l'avenir de la parole qui me vient? Il s'agit, je crois, d'un avenir entamé. La présente écriture partage déjà avec lui «sa personne fondamentale, qui est le je» (p. 7). Elle s'articule également depuis la «mémoire des lieux (livres, rencontres) où telle chose a été lue, dite, écoutée » : ainsi Barthes (p. 12) présente-t-il l'instance du je.

En ce sens, j'atteste du ton personnel qui vibre avec les mots. « Personnel», en considérant les desseins de l'élaboration courante: écouter un sentiment singulier, au vif de la rencontre avec l'œuvre d'art et construire une parole qui le donne à entendre. Écouter et entendre un sentiment singulier, en le laissant résonner avec les écrits des autres - sujet amoureux, artiste, dramaturge, historien, philosophe, psychanalyste. L'écouter et l'entendre, en résonance avec ce qui vit au-dehors, parmi les hommes.

Pour la petite histoire, c'est la recherche d'une œuvre d'art destinée au mur de mon bureau qui me mène à rencontrer un drapeau taliban. Combien est-il étrange d'ouïr le timbre de l'amour dans ce qui résonne depuis cette rencontre.

\section{Home}

La phénoménologie de Bachelard (1957, p. 25) désigne la maison comme « [l'] une des plus grandes puissances d'intégration pour les pensées, les souvenirs, et les rêves de l'homme» (p. 26). L'enceinte habitable établirait un «premier monde» (p. 26), et le philosophe explore les rêveries qui s'y rattachent. Il en livre le potentiel poétique.

Ma maison, la pièce du bureau, et là, un mur, inoccupé. C'est en cet endroit que l'écriture commence. Un chez-soi enracine le mouvement créateur. Le mouvement créateur altère l'habitation du chez-soi.

Selon ma compréhension, c'est dans un rapport particulier à l'espace que l'on accède à la maison librement pensée, remémorée, imaginée. L'imagination implique le travail d'une «volonté d'opposition devant l'espace affronté» (p. 206). En ce sens, par une formule lumineuse, Bachelard explicite «une puissance qui fait reculer l'espace, qui met l'espace dehors, tout l'espace dehors pour que l'être méditant soit libre dans sa pensée» (p. 207). Le fond poétique du logis prend forme, une pensée personnelle avance, quand la concrétude du monde s'éloigne, s'écarte, s'étrangéise.

Je m'apprête à raconter des rêveries, et des lecteurs la découvriront, avant même que soit détaillé le contact avec l'œuvre d'art. Elles sont introduites comme prémisses. Pourtant, chronologiquement, elles se présentent à moi 
comme résultats. Mon esprit se laisse captiver par des impressions émues, quasi divagantes, après la rencontre avec une œuvre. Après seulement, car au départ, le mur inoccupé ne me parle pas. Plat, utilitaire, insensible, il ne me dit rien, et je ne rêve pas. Alors je me mets en quête d'une œuvre à acquérir. Et quand je pars en quête d'avoir, je ne pense pas la perte, ni les différents visages de la perte. Or, une ouvre m'a éveillé, et c'est maintenant qu'une partie de la maison se dégage de la concrétude.

L'événement se déroule comme suit. Il y a un mur. Un jour, le mur recule, ouvrant l'aire du rêve. À partir des fragments d'un discours amoureux, le mur se recompose. Je me retrouve alors devant...

... un mur vide. Le mur transporte à l'arrêt d'autobus. «Enfant, je n'oubliais pas: journées interminables, journées abandonnées, où la Mère travaillait loin; j'allais le soir, attendre son retour [...]; les autobus passaient plusieurs fois de suite, elle n'était dans aucun» (Barthes, 1977, p. 21). Face au vide, je trouve «Absence» (p. 19). L'autre ne revient pas...

... un mur blanc. Le mur confronte à la présence déserte. Il vibre d'une «voix endormie, voix déshabitée, voix [...] de la fatalité blanche» (p. 131). L'indifférence tombe. «Personne ne répond, car ce qui est donné, c'est précisément, ce qui ne répond pas» (p. 133). Énigme du «Fading» (p. 129): devant, le retrait d'une âme...

... un mur fantôme, à l'instar du Vaisseau, dans l'opéra de Wagner. Le mur m’emporte, à la recherche du passager idoine. «Le Hollandais maudit est condamné à errer sur la mer tant qu'il n'aura pas trouvé une femme d'une fidélité éternelle.» (p. 118). La quête devient «Errance» (p. 117). L'absolu échappe...

Arrive-t-il que, dans l'espace de la maison, les murs ne s'éloignent pas? Absence, fading, damnation à l'errance: la perte et ses différents visages restent parfois impensables, inéprouvables, certes déjà là, en puissance, mais gardés hors de notre sensibilité. Quand le cœur résiste à la perte, il faut posséder, mur à mur.

D'où vient la puissance qui fait l'éloignement? D'où vient qu'un jour, j'aie pu rêver? 


\section{Origines}

La possibilité de rêver: destin possible d'une transmission, sinon d'une donation. Dans les termes de la psychanalyse laplanchienne, cela s'appelle aussi la séduction, et plus globalement, le primat de l'autre (Laplanche, 1987, 1990). Il rêve, comme on écrit: «Il y a».

Il rêve, puisqu'un autre a aiguillonné la rêverie. Il rêve, et je rêve, parce qu'un autre, avant moi, a rêvé. Je rêve, nous rêvons, quand l'autre attise non seulement une excitation, non seulement un sentiment, mais qu'il offre un genre d'étai permettant d'en penser quelque chose. Si un autre a su se montrer créatif envers la perte, envers les pertes.

Cependant, existe-t-il une perte qui soit fondamentale? Une perte qui ne se réduise pas à la disparition de l'autre, à son absence physique, ou à l'altération de sa présence? Lecteur de Laplanche, Scarfone (1994) porte à mon attention une perte transmise en même temps que la séduction. Une perte primordiale à laquelle chacun serait confronté, dès qu'il se retrouve face à un autre humain - à un «objet» humain. L'épreuve de la perte apparaîtrait au cour de la rencontre. «La perte est dans l'objet» (Scarfone, 1994, p. 99), conçoit-il. Ainsi, aucune nécessité que l'autre s'en aille pour le perdre. L'objet peut bien être là, à vue et satisfaisant au plan des besoins; néanmoins, il est déjà perdu. Aussi présent soit-il, il y a quelque chose de l'autre qui nous échappe, insaisissable. Ainsi, il existe une dimension de la réalité qui ne se restreint pas au monde perceptible, mais qui plutôt se présente avec l'autre humain.

Dans ce courant de pensée, l'insaisissable ressort de l'inconscient sexuel. Originairement, celui-ci compromet les messages que l'adulte communique à l'enfant. Plus largement, il infiltre les messages échangés entre les humains. En ce sens, le psychanalyste compare l'inconscient à un bruit, qui obscurcit les messages venus de l'autre: «C'est le creux au sein du plein, l'absence au cœur même de la présence» (p. 99.). Le bruit: la part déliée du message, ce qui, pour l'émetteur lui-même, n'est pas intégré au domaine du sens.

Éventuellement, quand j'arrive à Didi-Huberman (1992), une impression insiste: il n'est pas fortuit que mon mur s'éloigne, après (ou pendant) la rencontre avec une ouvre, et que, alors des rêveries défilent à la place d'un mur inoccupé. L'œuvre d'art, "porteuse d'une énergétique et d'une latence» (Didi-Huberman, 1992, p. 67), se reçoit-elle comme un message qui aiguille l'excitation et la symbolisation? Un message qui trouble, en même temps qu'il transporte suffisamment d'inhibition pour étayer l'imagination? Le philosophe ne réfère pas explicitement à Laplanche et à Scarfone. 
Néanmoins, le lisant, je repense à eux. À l'instar de l'autre de la séduction, les images de l'art confronteraient à une perte, susceptible de mettre la pensée en mouvement. Selon Didi-Huberman, l'expérience proposée par l'œuvre d'art détonne par rapport à l'expérience ordinaire de voir:

L'expérience familière de ce que nous voyons semble le plus souvent donner lieu à un avoir: en voyant quelque chose, nous avons [...] l'impression de gagner quelque chose. Mais la modalité du visible devient [...] vouée à une question d'être, quand voir, c'est sentir que quelque chose inéluctablement nous échappe $[\ldots]$ : quand voir, c'est perdre. (p. 14)

En outre, le philosophe conçoit les images de l'art comme les héritières des jeux de l'enfant: les jeux que celui-ci invente, et par lesquels il survit à la perte. La perte, ou l'épreuve dans laquelle quelque chose (lui) échappe.

Les images de l'art $[\ldots]$ savent présenter la dialectique visuelle de ce jeu où nous avons su $[\ldots]$ inquiéter notre vision et inventer des lieux pour cette inquiétude. [...] Les images de l'art savent en quelque sorte [prolonger] ce jeu de l'enfant qui ne tenait qu'à un fil, et dès lors elles savent lui donner un statut de monument, quelque chose qui reste, qui se transmet, qui se partage (fût-ce dans le malentendu). (p. 69)

À la lumière de cet extrait, nous pouvons comprendre l'objet d'art comme passeur d'une possibilité de jouer, de rêver, de penser. Possibilité émergeant de l'inquiétude qui cortège la perte. En effet, ces jeux d'enfants, que le philosophe met en correspondance avec les œuvres d'art, apparaissent intimement liés à l'épreuve d'une perte constitutive.

Didi-Huberman raconte ainsi des jeux d'enfants. À un niveau empirique, et dans les parages de ces jeux, apparaît la perte d'une figure investie, la mère. Il cite Freud (1920) et Au-delà du principe de plaisir: sur fond de guerre mondiale, une mère quitte la maison. Elle reviendra plus tard. Dans l'intervalle, son fils, tout petit, invente un jeu avec une bobine. «O-o-o-o!» s'écrie-t-il, quand il la fait disparaître, en la jetant au loin. «Da!» (ou «Là!», «Voilà!») s'exclame-t-il, quand il la fait réapparaître, en tirant sur le fil. Par l'intermédiaire de la bobine, on dirait que l'enfant remet en jeu le départ et la réapparition prochaine de la mère. Didi-Huberman cite également Fédida (1978) et L'absence: une mère est morte. Elle ne reviendra jamais. À leur tour, Laure et sa sœur, désormais orphelines, jouent à mourir. Encore et encore, 
elles se recouvrent d'un drap de lit, puis disparaissent. Éventuellement, les fillettes réinventent ce drap dont elles avaient fait un suaire: celui-ci devient une robe, une maison et un drapeau que l'on hisse, à la cime d'un arbre.

Mais ces jeux ne ressembleraient pas à des jeux s'ils n'étaient pas fondés par une autre perte: la perte de la plénitude, ou de la totalité du visible. Dans ce sens, le philosophe décrit une opération quasi miraculeuse accomplie dans ces jeux d'enfant: la bobine et le drap cessent d'appartenir uniquement au visible des volumes. Ils s'altèrent désormais; leur caractère utilitaire est surpassé. Ils font résonner autre chose, une inévidence qui ne se voit pas manifestement. Et la création de l'œuvre d'art commémorerait l'expérience de cette perte de totalité.

Cette autre chose, qui résonne dans la bobine et le drap, aurait rapport à une forme d'intériorité. "Un creux», écriront Laplanche et Scarfone. «Un vide, un dans, un dedans», écrira Didi-Huberman. L'intériorité évoque une dimension qui ne se laisse pas maîtriser par l'œil-organe, mais qui s'empare plutôt de «l'œil de l'esprit», pour emprunter une expression que Pontalis (1990, p. 56) tenait lui-même d'un peintre romantique. Un œil sensible à une dimension de la réalité qui n'est pas immédiatement perceptible. Nous admettrons que l'œuvre d'art éveille cet œil-là, déjà ouvert par les jeux de l'enfant. Un œil, peut-on ajouter, que les messages du monde adulte avaient entamé.

Didi-Huberman conçoit ainsi une dialectique, structurant l'expérience visuelle, notamment dans la rencontre avec l'œuvre d'art. Cette dialectique, il la nomme «l'inéluctable scission du voir». D’une part, l'enfant a vu: une bobine ou un drap. Ou, plus largement, formule-t-il, il y a «ce que nous voyons ». Mais, d'autre part, quelque chose a regardé l'enfant, à travers cette bobine et ce drap. Vraisemblablement, des états affectifs et des mouvements pulsionnels, peut-être une forme de désir pointant vers la mère, ont inquiété l'enfant dans la rencontre avec ces objets malléables. Dans ce que nous voyons, il y a aussi «ce qui nous regarde». En ce sens, Didi-Huberman (1992, p. 13) propose: "Chaque chose à voir, si étalée, si neutre soit-elle d'apparence, devient inéluctable lorsqu'une perte la supporte [...] et, de là, nous regarde, nous concerne, nous hante.» En excès par rapport aux images que nous voyons, quelque chose nous remue.

De façon radicale, je crois que l'auteur va jusqu'à suggérer que nous commençons à voir vraiment lorsque notre vision est aussi subjective, c'està-dire lorsque, voyant, nous sommes en même temps personnellement touchés, d'un sentiment qui ne se laisse pas saisir. Dans la foulée, il formule une invitation: 
Ouvrons les yeux pour éprouver ce que nous ne voyons pas [...] pour éprouver que ce que nous ne voyons pas de toute évidence (l'évidence visible) nous regarde comme une œuvre [...] de perte. (p. 14)

Dans la tentative de combler le mur d'une œuvre d'art, j'aurai trouvé à perdre.

\section{Cuvre}

J'ai parcouru des galeries en vain, à la recherche de l'objet idoine. Puis, un jour, flottant sur les liens internet, je me retrouve à la page de Miles Collyer. Je suis alors attiré vers les pièces qui composent une série, intitulée Home-made, Home-grown (Collyer, 2007). La traduction littérale: «Fait maison, de chez nous. »

Si nous explorons plus avant l'album des expositions (Collyer, 2006; 2007 ; 2008; 2010) auxquelles cet ensemble a pris part, on y voit « 6 handsewn felt flags» (Collyer, 2010), c'est-à-dire six drapeaux en feutre, cousus à la main. Ils sont fixés au mur ou à la fenêtre. Un nom est accolé à chacun: Al-Qaeda Organization in the Land of the Two Rivers, Hamas, Hezbollah, Jamaat Ud Dawa, Taliban, Chechen Republic of Ichkeria. Et sur les drapeaux, on reconnaît le signe respectif de ces groupes.

En outre, certains d'entre eux correspondent à des «entités terroristes » en vertu de la loi antiterroriste en vigueur au Canada (Sécurité publique Canada, 2016), notamment. Il s'agit de groupes armés et de factions impliqués dans des politiques internationales litigieuses. Ils participent à des conflits meurtriers, dont les enjeux idéologiques ou identitaires transcendent l'aspiration à posséder des territoires.

Éventuellement, à la lecture des essais de Hutton (2010) et de Heather (2011), la présence de distorsions retient notre attention. Les feutres d'une autre teinte se superposent aux teintes principales, créant la perception de limites floues. Embrouillement léger des emblèmes. En ce sens, Hutton observe: "Their vague appearance emphasizes their status as representations of representations, similar to their originals but twice removed.» (Hutton, 2010) La série Home-made, Home-grown présente des images d'images. Plus précisément, les étoffes qui la composent représentent des images pixélisées de drapeaux.

Les textes de Hutton et Heather nous apprennent ainsi que les drapeaux sont réalisés sur le modèle de photographies et de vidéos à basse résolution, circulant sur internet. À partir d'une technologie rudimentaire, des 
groupes marginaux diffusent leurs messages. Des drapeaux flottent, visibles à l'arrière-plan de l'action, souvent barbare. Ce que l'œuvre montre manifestement: un détail de ces images d'action, filmées par les groupes armés eux-mêmes, détail reproduit et figé par de nouveaux drapeaux.

On s'applique à décrire le contenu de l'œuvre et à identifier quelquesuns de ses référents. Mais les phrases plus haut, traitant du visible, diffèrent des phrases qui transcrivent le sentiment, dans le contact avec l'œuvre. Or, peut-il seulement s'articuler? Lyotard (1990, p. 45) répond: «Le sentiment est une phrase. Je l'appelle phrase-affect. Elle se distingue en ceci qu'elle est inarticulée.» Dans la perspective du philosophe, qui rejoint une certaine psychanalyse, la phrase-affect relève de l'infantia, une temporalité étrangère au discours articulé, temporalité du bruit, par lequel s'échappe et nous saisit quelque chose de l'autre et de soi-même.

Combien laborieux de transférer un bruit dans la langue, de faire parler le sentiment. Au moins, puis-je espérer que des mots le revêtent? Des mots, mes mots? "Jamais les mots, jamais mes mots, ne seront miens.» (Pontalis, 1984, p. 196) Mots, (me) viendrez-vous?

\section{Inquiétude}

Mots pour révéler le sentiment généré par le contact avec Home-made, Home-grown. Je les ai recherchés. Jusqu'aux retrouvailles avec une expression allemande, qui manque d'un équivalent dans la langue française.

«Das Unheimliche»: on y reconnaît d'ailleurs le titre original d'un texte de Freud (1919). L'éditeur de la version française traduit l'expression en longueur: "Ce qui n'appartient pas à la maison et pourtant y demeure.» (Pontalis, 1985, p. 7) Aussi, il soutient que l' «Unheimliche» désigne «ce qui est l'objet même de la psychanalyse». Quant au traducteur du texte freudien, Bertrand Féron, il conserve la traduction d'abord proposée par Marie Bonaparte: «l'inquiétante étrangeté». Chez Roustang (1976), la traduction est encore semblable et différente: «l'étrange familier». Mais comment cette locution, presque poétique, se déplie-t-elle? Et ensuite, comment éclairet-elle l'émotion provoquée par le contact avec l'œuvre?

À la lecture, nous comprenons que l'intérêt de Freud, envers un objet étranger familier, excède l'intellect. Il ne pose pas simplement un regard détaché sur la maison de l'homme. Il ne se contente pas de statuer qu'un hôte l'habite. Plutôt, il éprouve un sentiment d'inquiétante étrangeté, un sentiment dont il discute la survenue envers ce qui se rencontre dans la fiction et «dans la vie» (Roustang, 1976, p. 259) - y compris dans le travail 
psychanalytique. Un sentiment auquel il est également assujetti: «Je fus saisi [...] d'un sentiment que je ne peux que qualifier d'unheimlich.» (p. 239240) Il errait alors dans quelque quartier romain, et se retrouvait itérativement au même endroit, malgré ses tentatives de s'en éloigner.

Plus précisément, que dénomme le sentiment d'inquiétante étrangeté? Dans les termes de Corin (2010, p. 13), il qualifie «l'impression que l'on ressent lorsque, dans le monde extérieur, apparaît une chose dont on pressent la familiarité intime mais qui est étrangère au moi». Quant à De M'Uzan (1974, p. 156), il observe la ressemblance possible du sentiment d'inquiétante étrangeté avec une dépersonnalisation non pathologique. Dans sa théorisation, il s'agit d'une expérience de «saisissement» (De M’uzan, 1977, p. ix) plus ou moins soutenue, durant laquelle l'identité du sujet est ébranlée. Ce dernier se découvre vivant au-delà de ses limites habituelles, identifié de façon inusitée à des personnes, ou encore donne passage à des pensées déconcertantes.

Si l' «unheimliche» s'impose en objet de la psychanalyse, le sentiment de l'«Unheimliche» témoigne peut-être d'une expérience vivante de cet objet. En outre, ce sentiment révèle l'événement d'une tension entre un étranger intime et la maison de l'homme. Il atteste d'une épreuve de décentrement à laquelle convie la psychanalyse, épreuve que la terre des hommes et son travail culturel préparent et appellent déjà.

De nouveau, je les revois, ces drapeaux qui font œuvre. M'accompagnerat-on?

\section{Inquiétude (appliquée)}

La notion d'inquiétante étrangeté m'aide à penser l'affect que les drapeaux Home-made, Home-grown induisent en moi. Ainsi mettent-ils en présence une intensité à laquelle je pressens être lié. Je pressens qu'elle me concerne, et pourtant, l'intensité demeure indéfinissable. J'imagine arriver à m'en approcher, bien qu'uniquement à partir d'une dérive. M'abandonnant comme il se peut, je trouve la maison hors de ses murs clos.

Balisant la dérive, Freud observe:

Il s'agit du motif du double dans toutes ses gradations et spécifications, c'est-à-dire de la mise en scène de personnages qui, du fait de leur apparence semblable, sont forcément tenus pour identiques, de l'intensification de ce rapport par la transmission immédiate de processus psychiques de l'un de ces personnages à l'autre [...] de sorte que l'un participe au savoir, 
aux sentiments et aux expériences de l'autre, de l'identification à une autre personne, de sorte qu'on ne sait plus à quoi s'en tenir quant au moi propre et qu'on met le moi étranger à la place du moi propre - donc dédoublement du moi, division du moi, permutation du moi -, et enfin [...] de la répétition des mêmes traits du visage [...], actes criminels, voire des noms à travers plusieurs générations successives. (Freud, 1919, p. 236)

Le psychanalyste relie l'effet du double à un motif d'inquiétante étrangeté. On suivra désormais cette piste. Le double, comme signal de présence d'un étranger familier. Sans poser mot à mot la correspondance de cette citation avec le contenu rencontré, sa ligne générale prépare une vision de l'étranger convoqué par Home-made, Home-grown.

Remarquable, l'effet du double dans l'œuvre, ou plutôt dans l'espèce de symétrie qu'elle établit avec la réalité sociale. Hutton (2010) attribue d'ailleurs aux drapeaux tissés par Collyer la qualité de copie fidèle. Non seulement leur forme, mais aussi leur sous-titre, reprenant littéralement le nom des groupes armés, matérialisent cet effet.

Est-ce quétrangement, le sentiment de quelque disparition? Cela s'avérerait cohérent avec la perspective freudienne selon laquelle le double représente un signe «avant-coureur de la mort» (Hutton, 2010, p. 237), création qui, à l'origine de la vie psychique, sert à «se garder de l'anéantissement» (p. 237). Qu'elle révèle l'anéantissement ou qu'elle en délivre, indice de la précarité et de la survivance, la figure du double communique ainsi avec le danger (ou le désir?) de ne pas être.

Puis, le spectateur, moi en l'occurrence, tendant à se relier à l'œuvre comme à un double. L'effet qui point dépasse le registre strictement visible.

1. L'effet du double retentit dans une performance réalisée par l'artiste (Collyer, 2010a), et à travers le mythe historique qu'elle appelle. La confection de l'une des étoffes se déroule dans ce cadre. À la manière de Betsy Ross, l'artiste est assis sur une chaise berçante et travaille sur une pièce, durant la nuit. En outre, l'argument de la performance présente la couturière portée par la légende: on crédite Ross de la conception du premier drapeau, pendant la révolution américaine.

D'une part, une forme d'identification à cette femme semble structurer le concept de la performance. Évoquant les États-Unis d'un autre siècle, 
l'artiste brode, en direct, le drapeau d'un état révolutionnaire contemporain néanmoins dissous, la République tchétchène d'Itchkérie.

En outre, la figure légendaire de la couturière apparaît elle-même familière d'une forme de dédoublement. Selon Marla Miller (2010), il ne fait aucun doute que Ross a existé et qu'elle travaillait à titre d'artisane, en 1777. Toutefois, l'historienne postule l'impossibilité d'attribuer la création de l'étoffe à une personne particulière: "Craftswomen like her, usually employees of someone else's enterprise and the makers of objects that never bore labels, worked in anonymity.» (Miller, 2010, p. 4-5) La confection originale du drapeau américain engage un ensemble de protagonistes, anonymes. Or, dans le récit patriotique, érigé en réalité historique pour nombre d'Américains, le «moi» de la femme héroïsée condense le «moi» de tous les gens qui auraient participé à la création de l'emblème.

Le sentiment d'inquiétante étrangeté insiste. J'ai l'impression que des personnes, des espaces et des temps se substituent les uns aux autres. Le si lointain devient aussi le tout proche. Et moi, qui suis-je? «Moi», où es-tu? Et combien?

2. Quand je rencontre l'œuvre, la modalité du double teinte le lien auquel je suis obligé. S'initie un lien par le regard que l'œuvre présentifie, et qui me sollicite.

En rapport avec cette intuition, j'estime que la position du spectateur vis-à-vis de l'œuvre ressemble à la position de l'artiste vis-à-vis du référent qui l'inspire. L'un comme l'autre regardent ou, plus étrangement, l'un à la place de l'autre regardent. Ils regardent un drapeau donné à la vue. «The installation makes us consider what it means to look at something that is asking us to look at it», explique Miles Collyer $(2015$, p. 7) concernant la démarche de son exposition How Do You Surrender to a Drone? Dans son versant inquiétant, le «nous» dilue la séparation entre le moi et l'autre, entre les spectateurs et l'artiste: il suggère une identité entre l'expérience de l'artiste et l'expérience à laquelle ce dernier engage le spectateur.

Exagérant encore les réverbérations du double, je me demande si, par exemple, quand le spectateur rencontre le drapeau taliban, il prend la place de l'œil qui a initialement filmé son image. Image home-made, produite par le groupe armé lui-même. Drapeau-portrait, image qui s'apparente tout aussi bien à un selfie groupal qu'à l'ego-portrait: si l'on admet l'identité de l'unité qui filme avec celle qui est filmée.

Le sentiment d'inquiétante étrangeté s'amplifie. Je contemple les drapeaux de l'œuvre et me situe à la place de l'artiste, apercevant des drapeaux 
sur internet. Mais pas uniquement. Je me situe à la place de l'unité terroriste, qui se fixe elle-même. Or, je me trouble à penser que, dans les situations s'apparentant à celles des autoportraits, personne ne se tient derrière la caméra. Qu'un doigt appuyant sur le détonateur, ou une activation automatique pré-programmée. Alors, peut-être, quand je regarde les drapeaux, je me retrouve à un lieu correspondant à un non-lieu: vide, impersonnel, désincarné. Depuis lequel personne ne voit. Depuis lequel «voir» ne voit rien. De cette place-là, l'image ne porte aucun mystère.

Si je m'y trouve, y a-t-il là quelqu'un, ou personne? Ou encore, figé là, estce que je n'existe plus en tant que personne? Impersonnalisé? Non-vivant?

3. L'inquiétude n'est pas finie.

M'imaginant confondu à l'œil automatique du selfie, capteur du drapeau-portrait, il ne me reste qu'un pas. De cet œil, je me vois. Me self-vois-je? Suis-je en train de regarder vers moi? En suis-je à guetter la présence de mon être à travers ces drapeaux?

Par réflexe, je personnalise trop vite ce lieu impersonnel. Car posté en un non-lieu, voit-on? Peu importe ce qui surgit devant, rien ne me regarde. L'intériorité n'existe pas. Voir sans me reconnaître. Surtout, voir sans la sensation de voir, sans le sentiment: comme mort.

On suppose une demande: «Look at me.» Depuis l'œil automatique, aucune pensée pour la demande: que répéter, que reproduire, portrait après portrait. Ou plutôt, soustrayant la notion de temporalité; répéter, reproduire portait sur portrait: drapeau, drapeau.

\section{Curieuses visions.}

Par moment, j'y entends l'allusion à une pensée qui, parfois, surprend l'esprit: «Si j'étais mort...» De M’Uzan (1974) interroge cette supposition ${ }^{1}$. Cela l'amène à considérer l'expérience d'un double: un homme se demande s'il est mort. Il recherche chez un autre, assis devant lui, les traces de sa propre vie, qu'il imagine disparue. Momentanément, l'homme fait de cet autre un double. L'expérience se déroule à la manière d'un fantasme éveillé, qui n'est pas dépourvu d'émoi.

Le psychanalyste comprend alors le phénomène dans un rapport de parenté avec l'anticipation d'un deuil. La construction d'un double répondrait à une menace d'anéantissement, réelle, pesant sur l'objet d'amour, fortement investi: la mère qui vieillit, dépérit, appelée un jour à mourir. 
Le phénomène est comparé à un compromis entre deux mouvements: «Le sujet exprime en même temps sa tentation de s'anéantir avec l'objet - en quoi il cherche $[. .$.$] à réaliser une introjection - et son effort pour se sau-$ ver en s'arrachant en tant que double à la sphère maternelle» (De M'Uzan, 1974, p. 161). Ces deux mouvements se relancent désormais. À l'instar des personnages de l'étude portée par De M’Uzan, le sujet est identifié à un fils et l'objet, à une mère.

D’une part, «si j'étais mort...»: le fils éprouvant l'incertitude de son existence trahirait un mouvement d'incorporation. Il confondrait l'image de lui-même avec celle de la mère vacillante. Il retiendrait et conserverait la mère à travers l'inquiétude de son sentiment d'exister. D'autre part, si l'homme, devant, était «moi» vivant, le fils, apercevant en face l'impression de sa propre vie, trahirait la tentative de se mettre lui-même au monde, en évitant la mort. Vis-à-vis le dépérissement de la mère d'amour, le fils lui-même se heurterait au danger d'être anéanti - ou, plus précisément, la représentation du fils, à l'intérieur de l'image qu'il a de sa mère. Des maisonsgigognes: la mère habite le fils, et dans cette mère intérieure, une image de soi que le fils investit. Quand l'ombre de la précarité recouvre la personne aimée, l'impression de symétrie viserait à restaurer cette image (l'image de soi dans la mère intérieure).

Devant des drapeaux qui ressemblent à s'y méprendre aux drapeaux de groupes terroristes, le sentiment de mon existence devient incertain. Dans l'écriture par laquelle ces drapeaux sont fréquentés, l'incertitude s'intensifie. Avec De M'Uzan qui explore la supposition d'être mort, le sentiment d'incertitude est transposé dans une langue du deuil. La recherche d'un double y révèle le danger de la disparition de la mère investie, en même temps que le risque de la disparition imminente d'une partie de soi.

Si seulement j'étais tout construit autour de l'amour pour les autres, même s'il est bouleversant de risquer de les perdre! Encore des pensées viendront, me rappelant à mon "hétérogénéité», selon l'expression de Quinodoz (2002). Cette cohabitation, au sein du fonctionnement psychique, de mécanismes ressortis à différents registres, des plus secondaires aux plus archaïques. Leur disharmonie peut faire craindre qu'on soit fou.

\section{Spectre}

Des pensées viennent. Certaines demeurent, retravaillées par le texte. Tant d'autres passent, dissolues par je ne sais quelle destruction. Au fait, disparaissent-elles vraiment? Ou changeraient-elles seulement d'habit pour 
révéler le sentiment, sinon autrement, le camoufler? Parfois reviennent des pensées, précédemment écartées.

Une impression fait retour et vient saisir l'esprit à nouveau. Impression spontanée, vive et dérangeante, apparue instantanément au contact de l'œuvre d'art. Depuis lors, un travail plus intellectuel l'a peut-être assagie. Cependant pas effacée. Il l'aurait plutôt diluée, voire obscurcie, à travers cette vision de l'œuvre dans laquelle je guette la présence de mon être.

Une impression fait retour: Je suis identifié au terroriste. J'écris ainsi, combattant l'idée suivante: Moi, terroriste.

Le titre de l'œuvre est reçu comme une interpellation qui nous désigne en tant que violent, talibans ou extrémiste. Interpellation qui me désigne.

Home-made. L'interpellation vient-elle du dehors ou du dedans? Je l'entends venir, nue. Un genre de folie opère, allant jusqu'à pressentir un vacillement dans l'épreuve de la réalité. Car, relâchée, l'épreuve du sens des mots: à ce moment, j'entends et je lis Home-made comme s'il signifiait «ici» ou «moi». Je me retrouve imputé de la violence. Or, selon une lecture fidèle, l'expression home-made dénote plutôt une production réalisée dans les limites d'un espace précis. Elle renvoie aux limites d'un espace précis et non à l'espace précis où l'on se trouve. Par le malentendu, le drapeau devant moi devient aussitôt mon emblème, et je porte le nom du groupe auquel il est associé. Des hommes violents sont (comme) moi et je suis (comme) eux.

Je succombe alors à la force de l'œuvre, force neutre dont le travail n'est pas de condamner les groupes associés à une violence extrême. Force à contre-courant de l'interdit de séjour que nos territoires leur opposent. Mais à contre-courant aussi de la propagande par laquelle les groupes armés répandent leur message: ici, nul acte d'enrôlement.

Force d'émanation, qui donne à imaginer, ou force «auratique» dirait peut-être Didi-Huberman (1992, p. 105). Par là, le philosophe souligne le pouvoir de l'œuvre à engendrer «ses images, ses images en constellations ou en nuages, qui s'imposent à nous comme autant de figures associées, surgissant, s'approchant, et s'éloignant». Ses images: c'est-à-dire des rejetons de la mémoire qui, au-delà de la visibilité de l'œuvre, désorientent l'esprit du spectateur lorsqu'il la rencontre. Ici, la mémoire ne se réduit pas à un temps linéaire, ou à la remémoration d'un souvenir: elle englobe jusqu'au fond inconscient à partir duquel celui-ci émerge. Mon malentendu illustre-t-il un archipel, parmi l'étendue des images que l'œuvre inspire?

Force humanisante, par ses effets. L'œuvre m'ouvre effectivement à une épreuve de la proximité et de la ressemblance avec l'étranger qui nous 
apparait habituellement terrible. D'ailleurs, et sans qu'elle ne s'y réduise, des aspects de l'œuvre soutiennent peut-être cette épreuve de la familiarité, jusqu’à son exagération en un malentendu.

Par exemple, dans le titre, le signifiant «home». Et la domesticité poétisable qu'il connote. Ensuite, la patience du travail artisanal, le temps pris par l'artiste à reproduire de ses mains, par sa chair, un symbole qui appartient pourtant au monde étranger. Aussi, doublant le visage singulier de chaque drapeau, le caractère quasi universel du drapeau lui-même, en tant que signe de ralliement. Enfin, la trace du cyberespace et l'ombre du selfie sur les drapeaux confectionnés par l'artiste: le monde civilisé n'utilise-t-il pas massivement internet pour partager des portraits?

Poussé par la force de l'œuvre, et suivant le chemin qu'emprunte l'écriture, je me déplace alors à travers un «spectre d'identité». En termes métapsychologiques, De M'Uzan introduit cette notion:

Si je n'est pas dans le Moi, il n'est pas non plus entièrement dans l'autre, mais réparti tout au long des franges d'un spectre, disons d'un spectre d'identité, défini par l'ensemble des diverses positions dont la libido narcissique est susceptible, ou plus précisément par les lieux et les quantités où s'investit la libido narcissique, depuis un pôle interne jusqu'à un pôle externe qui coöncide avec l'image de l'autre. (De M'Uzan, 1974, p. 163)

Ainsi, le «je» ne se réduit pas à une image fixe. Il a «la remarquable propriété d'errer sans se perdre à mi-chemin du dehors et du dedans» (p. 163). Spectrale, l'identité excède les limites du «moi»: à mon insu, des parties de mon âme sont déposées dans l'autre. Elles sont parfois retenues dans l'image que j'ai de l'autre et dans la vision que l'autre (tel que je l'imagine) a de moi. L'autre, comme "un avant-poste du sujet» (p. 163); l'autre, comme un horizon dans lequel je suis peut-être déjà, en position d'extra-territorialité.

Humanisante, la force de l'œuvre, dans la mesure où, par ses effets, elle élargit le sentiment de l'identité, jusqu'à l'image d'un étranger terrifiant. La force incite-t-elle à se perdre et à se trouver, et à se perdre et trouver encore, dans la fréquentation de cet étranger que l'œuvre porte jusqu'à soi.

Depuis l'aura de l'œuvre et de mon inquiétante identification, d'autres pensées se déploient; des visions prenantes, autres que celles de moi-même, par lesquelles la mémoire qui m’occupe se terre et se révèle. Là, l'intensité. 


\section{Pérégrinations}

Il était une fois, l'hiver. Pour les vacances, je souhaite voyager dans un territoire qui a connu la guerre. Une guerre pas complètement morte du champ de vision, dont il resterait suffisamment de marques à ciel ouvert. Qui n'a pas fini d'être refoulée, reconstruite par la mémoire des musées.

Je visite la Croatie, la Bosnie, l'Herzégovine et la Republika Srpska, région en conflit durant les années 90 . Conflit dévastateur dans lequel les opposants se voient dans un rapport de pure étrangeté en même temps que d'absolue ressemblance. Dans un reportage de la CBC, un soldat croate (ou serbe) s'exprime en ce sens à un journaliste (Scarfone, 2007, p. 217-218). D’un côté, il lance: "Nous nous battons pour un pays bien à nous, parce que nous n'avons rien de commun avec ces gens-là. Ils nous sont totalement étrangers.» Mais de l'autre, il affirme: "Nous savons parfaitement comment pensent ceux d'en face, nous les connaissons bien, ils pensent exactement comme nous, nous sommes pareils.» Je n'ai découvert ce soldat qu'après mon voyage.

À la fin de mon séjour, j'en entends néanmoins un autre, dans les archives. Je regarde l'enregistrement d'une audience, à la Cour internationale, où un soldat serbe est interrogé. Je traduis en français les paroles dont je me rappelle:

L'AVOCAT — Combien de musulmans avez-vous tués?

LE TÉMOIN - Je ne m'en souviens pas.

L'AVocat - Mais quand même. Combien de musulmans avez-vous tués?

Pouvez-vous donner un nombre approximatif? Combien, environ?

LE TÉMoIN - Je ne m’en souviens pas. J'ai oublié. Je ne le sais pas. Je ne m'en souviens pas. En fait, je ne l'ai jamais su. Je ne m'en souviens pas. Je ne veux pas m'en rappeler.

Est-ce parce qu'il pleure durant son témoignage, mais il me touche. Est-ce par ce qu'il dit, ou par ce qu'il ne dit pas? Comment survivre aux meurtres commis par soi? Je suis touché en imaginant le mal qu'il s'est infligé à lui-même, à travers ces meurtres innombrables, ou incalculés. Mais un mal à retardement, un mal absolument sans conscience au moment de la blessure. En écho au soldat précédent, dont les victimes étaient dites «pareilles» à lui: est-ce aussi sa propre mort qui est souhaitée à travers celle de chaque musulman abattu? Une part de lui est-elle exterminée, en même temps qu'il tue un autre? Part étrangère, que je me figure interdite à la familiarité? 
Des lectures queer reviennent alors, imprégnées de ce témoignage. «Le rectum est-il une tombe?» Léo Bersani (1987) formule cette question en titre d'un ouvrage. Il y décrit une sexualité à partenaires multiples, en série, à risque, dans un contexte anonyme. Les pratiques de "barebacking» - "engaging in unprotected sex» explique Bersani (2008, p. 64) - peuvent inclure jusqu'au dessein de contracter ou de transmettre le VIH volontairement. Quand l'innombrable se manifeste dans le contact sexuel, l'interrogatoire se réécrit:

L'Avocat — Combien d'hommes avez-vous baisés?

LE TÉMOIN - Je ne m'en souviens pas.

L'AVOCAT - Mais quand même, combien, environ?

LE TÉMOIN - Je ne m'en souviens pas. Je ne l'ai jamais su. Je ne veux pas m'en rappeler.

Il me touche aussi, ce témoin fictif, amant en série. Par analogie, je l'imagine poussé dans un manège qui répète l'anéantissement. Dans l'analyse de Bersani, l'extrême de la destruction est aussi précisé, à la manière d'une dispersion du sujet:

The barebacking bottom enters into an impersonal intimacy, not only with all those who have pumped their semen into his body, but also with all those unknown partners, perhaps now dead, with whom he has never had any physical contact. His subjecthood is, we might say, absorbed into the nameless and faceless crowd that exist only as viral traces circulating into his blood and maybe fatally infecting him. (Bersani, 1987, p. 93)

Un tort est-il commis envers le "visage», cela qui «nous interdit de tuer» comme l'écrit Lévinas (1982, p. 80), cela qui impose que «Toi, c'est toi» (p. 81), et qui singularise l'autre humain, mon prochain.

La vision d'un visage disparu montre aussi la destruction, le sexe, et une forme de passion qui se condensent dans la pièce de théâtre intitulée Being at Home with Claude. Yves, un prostitué, est interrogé par un inspecteur, pour le meurtre de Claude, son amant. Yves s'exprime par mots courts ou phrases brèves durant la première partie du texte. Puis, quand il prend pour de bon la parole, il entame un long monologue.

Vous savez, les histoires nounounes de: lui c'est moi, moi c'est lui? C'est vrai. Ça existe. J'sais pas comment expliquer ça. Mais c'est ça. J'avais pas 
l'impression de t'nir quelqu'un dans mes bras. J'avais pas l'impression qu'y'avait une différence ent'lui pis moi. (Dubois, 1986, p. 103)

Il raconte ses derniers ébats, "at home», avec l'homme qu'il dit aimer et qu'il a tué en baisant. Son récit se termine par l'aveu d'un suicide en filigrane de l'homicide:

J'vous ai raconté tout'ça parce que là, y'a pus rien qui a du sens. Y'a rien que lui. Le souvenir de lui, qui a un sens. Même si j’y ai pas pensé, en mots, j'sais qu'en l'tuant, j'me tuais moi avec. Pis c'est ça que j'voulais. Mais en attendant ma vraie mort à moi, lui, y vit en moi. Comme personne l'a connu. Rien que moi. Y est rien qu'à moi. Mais. Là, c'est en train de tout'se défaire. (p. 115)

«C'est en train de tout'se défaire»: il y a une quantité de meurtres, d'amants, de mots. Et là, une fragilité.

L'indice de la quantité annonce peut-être combien je suis, combien nous sommes, «écrivants» artistes, amants réels ou imaginaires, soldats, terroristes, destructibles, fragiles, et malgré tout bien vivants. Mais d'une vie qui, sous l'emprise de la quantité, ne serait pas librement vécue.

\section{Quantité}

Tuer, baiser, écrire, compter l'incomptable des visages disparus: la quantité des meurtres, des amants, celle des mots peut-être même, quantité manifeste et factuelle, fait-elle écho à une quantité, celle-là plus obscure, poussant depuis les profondeurs de la vie humaine?

Depuis qu'un discours amoureux sort de mes murs, ma contenance s'effrite par intermittence. Voilà maintenant que je foule la Bosnie, me recueille sur la tombe de corps vivants ou morts entre-pénétrés, et m’immisce dans la maison de Claude. Le parcours vécu s'emmêle dans une impression de violence et de perte de sens. Sans dedans dehors, une voix (me) demande: en rapportant ces pérégrinations, que vouloir dire? Et que signifient les actes de tuer, baiser et écrire, certes respectivement différents, qui se relaient?

Classiquement, au chapitre des forces profondes, la psychanalyse postule l'action de pulsions participant à la surdétermination de nos conduites. Ces pulsions, certains théoriciens les qualifieront entièrement de sexuelles. Dans cette lignée, Laplanche (2003, p. 151) demande: «Quelle est la part de sexuel dans tout crime, fût-il le plus ordinaire, le plus banal, le plus réaliste?». Il 
met alors en cause un désordre interne, celui d'une sexualité infantile non liée, d'un inconscient abrupt et mortifère participant au crime.

Rappelons-nous le bruit qui excéderait le message humain. Le sexuel délié du crime s'apparenterait ainsi à ce bruit, mais avec un contrepoids extrêmement minimal du côté de la symbolisation et de la tempérance: «la bêtise de l'inconscient», désigne Pontalis (1997, p. 118). En toute fulgurance, elle «tient l'intelligibilité en échec» (p. 119). Un fragment de réel qui ne serait pas rêvé dans une rêverie, et qui pousserait à l'action. Et s'il y avait rêverie, il y persisterait un point aveugle. Le fragment de réel non-rêvant orienterait néanmoins la rêverie depuis quelque profondeur.

À l'instar d'Arendt (1971), si l'on rapproche le mouvement de la pensée avec celui d'un vent destructeur, un vent autodestructeur même, concevra-ton que le spectre du crime travaille également l'écriture? Crime, avec ce qu'il porte d'excès, de déliaison, de sauvage; crime nécessaire, fructueux? Qui sait? Destruction sûrement distincte, néanmoins, par le tort qu'elle fait subir au familier, de celle d'une écriture périmant l'étrangeté de l'affect. "Tout crime», cela inclut peut-être jusqu'à l'acte commis par l'écriture, lorsqu'elle œuvre à défaire le déjà pensé, à l'éloigner et lorsqu'elle rature aussi, impitoyable, ce qu'elle a elle-même déjà écrit.

Dans «tout crime», si ce n'est menant au crime, la pensée aurait donc échappé quelque chose. Ou quelque chose lui échapperait, infiniment. La conscience personnelle n'aurait pas intégré une part d'âme: une trace excitante gravée en un recoin impénétrable de soi, du monde, et qui commanderait nos actions. Trace gravée depuis l'enfance, archivant ce que le petit être a échoué à traduire des messages que les adultes lui adressèrent. Gravée, ou résultant d'une «implantation », voire d'une «intromission» (Laplanche, 1990, p. 358), quant au procès du message qui s'impose violemment, « rebelle à toute métabole» dans la chair de celui qui le reçoit. Trace susceptible d'être ranimée par les rencontres subséquentes avec les autres humains.

Dans ce contexte, les adultes et les autres humains évoqués ici ne désignent pas une figure abstraite. Ils prennent part aux communications marquant singulièrement chacun, parfois jusqu'à subjuguer. Messages rencontrés d'abord dans l'environnement nourricier, à travers celles et ceux que l'on appelle généralement (grand-) mère, (grand-) père, sœur, frère. Ensuite, tant de messages hétérogènes investis dans l'après-coup, transmis par le chœur social discordant des pairs citoyens qui, par mots et gestes, (me) parlent. Au sein de cet ensemble, "écrivants», soldats, artistes, amants, terroristes, sont susceptibles de prendre place. Nous y prenons place. De proche ou de loin. 
Car dans notre monde technologique, le message de l'autre peut arriver du lointain, traversant internet et les réseaux dits «sociaux». Cybermessages qui raniment la trace du pulsionnel et les messages inassimilables, déjà en soi.

Par ailleurs, suivant des développements complémentaires, De M'Uzan (1984, 2004, 2005) envisage le déferlement d'une autre forme d'énergie, celle-là absolument contraignante, en deçà de la pulsion sexuelle: «Une excitation pure, avide d'être déchargée par les voies les plus directes» (De M’Uzan, 2005, p. 149), une quantité sans qualité sexuelle. Dans un texte précédent, il l'avait déjà rapprochée d'un «instinct biologique, hors d'état d'être pris en charge par l'appareil psychique pour devenir pulsion» (De M’Uzan, 1984, p. 163). Cette énergie concernerait en quelque sorte le corps brut, proto-humain, doublant la rencontre avec l'autre de la culture; le corporel n'établissant pas de seuil avec le psychisme, ou ayant rompu avec lui, un genre de corporel délié.

Le psychanalyste appelle alors «esclaves de la quantité» les personnes dont le comportement serait contraint par de tels accès d'excitations sans qualité, faisant incessamment et irrésistiblement retour. "Écrivants», soldats, artistes, amants, terroristes: en sommes-nous - en suis-je - de ces esclaves?

Dans sa potentialité, le statut d'esclave tiendrait d'un ordre universel: «Il y a en chacun comme un germe, une virtualité intrinsèque d'asservissement aux excitations puissantes.» (p. 165) Cependant, De M’Uzan trouve plus spécifiquement la manifestation de cette servitude à la quantité dans les passages à l'acte violents et dans les passages à l'acte sexuels dits "pervers », bien souvent empreints de violence. Éventuellement, il repère la servitude à la quantité à l'aune des activités relatives à la créativité.

Ainsi, l'artiste, l'écrivain, et plus largement le créateur, par leur travail forcené, un genre d'addiction au travail, sont nommés « esclaves de la quantité par défaut» (De M’Uzan, 2004, p. 135). Selon cette conceptualisation, «une véritable carence existentielle, un défaut d'être, une incapacité à se sentir naturellement soi-même» présente la quantité pour corollaire. Dans ses racines vitales, pré-libidinales, la créativité réagirait à «une défaillance du tonus identitaire de base» (p. 139). Le petit démuni d'hier rechercherait à parer cette insuffisance exaspérante à travers la dilatation de soi procurée par la création. Il serait mû par l'attrait d'un genre de grandeur paradoxale, en quête de toujours plus que ce qu'il trouve simplement en lui.

Le vent destructeur qui relance la pensée, s'il est entretenu par du sexuel délié, souffle-t-il aussi depuis une blessure profonde? Blessure incompatible 
avec la tranquillité du familier et avec des limites sans équivoque. Blessure qui exigerait, de la part du créateur, qu'il amplifie encore toujours plus son être, en défaisant et en recomposant les formes connues. Blessure qui le presserait à s'aventurer vers l'ailleurs, en s'y perdant encore plus loin.

Selon ma compréhension des écrits de De M’Uzan, la quantité, «en excès» ou «par défaut», concerne donc une blessure identitaire primordiale. Blessure archaïque, qui remonterait à "un temps où l'être humain est physiologiquement très mal armé» (De M’Uzan, 1984, p. 164). Ce temps ressort de l'accouchement et de la confrontation infantile au monde humain, qui succède au corps de la mère. Temps d'une extrême passivité, dans laquelle les petits êtres se trouvent radicalement démunis, leur corps tout entier livré à des pressions considérables, à des excitations perturbatrices dépassant un seuil de tolérance génétiquement programmé. Détresse et désespérance: comme si d'aucuns se remettaient suffisamment de l'épreuve d'être né, de la traversée de la première frontière, vers la rencontre avec l'autre.

"L'état de détresse sera prêt à se reproduire à n'importe quel moment» (p. 162): blessure revenante donc, menaçant sans cesse de son imminence, entravant l'intégration du monde pulsionnel. Les pulsions, susceptibles de devenir excessives, pourraient raviver la vulnérabilité originelle: «Excès libidinal qui, du fait même de cet excès, peut provoquer [la] dégradation qualitative de la pulsion en direction (à l'extrême) d'une énergie d'excitation que seule la décharge va pouvoir gérer» (De M’Uzan, 2004, p. 136-137). La blessure est abrasive, le sexuel s'y défait, et ce, même si l'acte passe manifestement par la sexualité. Si la psychanalyse interroge la part du sexuel dans tout crime, la question se transforme désormais: que reste-t-il du sexuel dans l'acte du crime? Et dans l'acte d'écriture?

Selon les théories que nous avons feuilletées, le tracé depuis la Bosnie jusqu'à la maison de Claude convoque vraisemblablement du sexuel délié, résistant au sens, dans l'impasse à traduire certains messages d'un chœur social discordant. En deçà de la «ligne du sens» (De M’Uzan, 2005, p. 150) ou en rupture radicale avec la question du sens, une quantité déqualifiée, effet d'une blessure fondamentale, viendrait aussi de concert avec les pérégrinations précédentes.

La voix qui (me) demandait du sens devra alors attendre, à moins qu'elle ne mue. Et moi avec elle.

Une voix demande, sans attendre, l'émoi avec elle; voix demande émoi. 


\section{Mots parmi les mots}

La présente écriture se compose à partir de détournements. Encore une phrase s'est éloignée, n'est plus comme elle était. Surtout, une phrase arrive. Autre, elle dévie la trajectoire des mots, des lettres et des sons déjà arrivés. Elle vient s'y superposer, introduite en relief par un caractère italique, avec ses lettres inclinées vers l'avant. Lettres dont la forme illustrerait la force des phrases qui poussent jusqu'à l'incursion dans la maison du soi, jusqu'à l'intrusion au sein du texte. Phrases heurtant la tranquillité d'une compréhension monotone.

Scarfone (2012) nous sensibilise à la possibilité d'une telle force, lorsqu'il rapproche l'inscription de paroles sur des peintures qui présentent l'Annonciation, avec la survenue d'éléments discursifs dans les rêves. Par là, il étudie les limites de la représentation et suggère que des mots, sur les images de la peinture ou du rêve, indiquent une ratée dans l'accomplissement de désir. Malgré un travail de symbolisation rendant possible l'image, le travail psychique échouerait à digérer ou à transformer un contenu traumatique pour le représenter en une scène intérieure, visuelle. Si l'élaboration par l'écriture comporte des affinités avec le rêve, ses mots peuvent-ils aussi révéler la pointe d'une défaillance? D'ailleurs, sous la perspective d'un accomplissement de désir, Freud (1908) relève une correspondance entre la création littéraire, la fantaisie et le rêve nocturne. Rêve et création discursive partagent-ils alors un pouvoir excédentaire, celui de reporter un péril plus ou moins partiel, glissant dans le texte sous les allures de mots parmi les mots?

Ces paroles particulières, Scarfone (2012, p. 71) les entend donc à titre «d'exclamations, nullement discursives». Malgré leur apparence verbale, et au-delà de leur sens linguistique, reconnaissable, elles compteraient plutôt pour l'étranger, l'obscur, qu'elles font apparaître. Selon le psychanalyste, «elles trahissent une surcharge, la présentification d'une irruption, possiblement traumatique, dans la texture même du rêve [ou de la peinture]» (p. 91). «Irruption de l'invisible dans le visible» (p. 85), «venue de l'infini dans le fini» (p. 84): les phrases, transcrites en italique dans le texte ci-écrit, émergent-elles depuis un dépassement semblable?

Dépassement des mots par une intensité qui les déborde et qui ne finit pas de déborder, non seulement à travers les mots eux-mêmes, par des mots factices, mais aussi par le triomphe des actes qui bouleversent l'ordre du monde. Tuer, baiser, déshumaniser, écrire. L'écriture transporte-t-elle aussi l'excès, révélé par des «mots» devant lesquels je suis démuni? Simulacres de mots, signifiant une affectivité aiguë, une blessure vitale, parmi les mots 
d'une langue intelligible, et l'intelligibilité, que j'espère construire en articulant, n'épuiserait pas la sonorité étrangère des messages traumatiques qui me regardent dans ce que je vois, rêvant éveillé.

Ces derniers peuvent se relater ainsi : l'intitulé Home-made désignant des drapeaux en forme de terrorisme, le nom terrifiant respectivement accolé à chacun de ceux-ci, les blessures d'amour qui se mettent à resurgir depuis un mur inoccupé, l'œil automatique depuis lequel je me self-vois (sans me reconnaître), le discours intérieur disant le sentiment d'être interpellé par le mal, l'interrogatoire incriminant d'un avocat envers un témoin, soldat ou amant, le titre «at home» pour une pièce de théâtre violente, et maintenant, l'impératif d'être ému brisant la phrase qui précédait.

Sous l'emprise de telles paroles qui n'en sont peut-être pas, ou qui seraient aussi autre chose, je travaille néanmoins. Suivant la poussée des mots saisissants, comme si c'est tout ce que je pouvais faire. Leur offrant conflictuellement mon corps, à l'occasion insomniaque, pour qu'ils existent. Sillonnant tant bien que mal le spectre de l'humain dans les résonances de leur aura.

Le travail actuel tire à sa fin. Temporellement, nous en sommes bien après le contact empirique avec l'œuvre d'art. Pourtant, intellectuellement, je continue à m'enquérir: que s'est-il passé? Je cherche des pistes pour comprendre comment du pensant et du traumatique se sont mis à croiser la trame des phrases qui me viennent, depuis une rencontre avec un drapeau taliban. Encore: d'où vient que, un jour, j'aie pu rêver? Rêver avec l'excès et le défaut qui précipitent la pensée. Penser en dépit du péril qui se perpétue en rêvant, qui (se) transforme peut-être aussi. Est-ce l'effet d'une voix en demande d'émoi, mais actualisant cette recherche, je me reporte intuitivement au discours amoureux.

Historiquement,

c'est la femme qui donne forme à l'absence, en élabore la fiction, car elle en a le temps: elle tisse et elle chante; les Fileuses, les Chansons de toile disent à la fois l'immobilité $[\ldots]$ et l'absence (au loin, des rythmes de voyage, houles marines, chevauchées). Il s'ensuit que dans tout homme qui parle l'absence de l'autre, du féminin se déclare: cet homme qui attend et qui en souffre, est miraculeusement féminisé. Un homme n'est pas féminisé parce qu'il est inverti, mais parce qu'il est amoureux. (Barthes, 1977, p. 20) 
«Elle tisse et elle chante». J'imagine du coup l'artiste, Miles Collyer, avec ses fils et ses tissus. Je me demande s'il chantait durant le processus créateur. Je le lui demande. Est-ce une façon de prêter l'oreille à quelque fond sonore de la série Home-made, Home-grown? Une façon oblique d'y sonder le signe d'un amour en attente. Au fil de notre correspondance irrégulière, je l'interpelle donc:

- Did you sing?

- I did not sing. I've never sung and rarely hum or whistle in my daily life.

Il m'écrit plutôt l'état calme et contemplatif que la couture lui inspire. Il décrit son silence pendant qu'il se concentrait à la tâche, accompagné par le flottement de ses pensées. Puis, il qualifie le mouvement accompli par son travail sur les étoffes: "returning the photographic image to a tangible textile object». L'idée suggère un retour, un rétablissement.

S'agit-il d'une régression de l'image vers l'expérience de la tactilité, qui lui serait antérieure? Dans cette lignée, Freud remarque d'ailleurs (1905, p. 90) que la vue est «dérivée en dernier ressort de l'attouchement». En outre, dans ce travail de couture et de superposition, s'agit-il de restituer une base sensible matérielle et muable à l'image virtuelle qui en est dépourvue? D'animer une surface de contact, de passage et d'empreinte? Une telle interface, ne l'appelle-t-on pas aussi maison et murs, corps et peau?

Les propos de l'artiste m'amènent à considérer la sensorialité, la sensibilité, en jeu dans l'œuvre, de même que le caractère primordial de cellesci pour le psychisme. En ce sens, De M'Uzan (2015, p. 234) souligne que «la perception correspond à un temps fondamental dans l'installation du fonctionnement psychique». Il nomme «vital-identital» l'ordre qui serait immédiatement engagé dans la perception, tout en négociant avec l'ordre du «sexual». La blessure qui nous a précédemment intéressé, celle-là qui fait courir la quantité sans qualité et fait obstacle à la mentalisation du sexuel, serait d'ailleurs située à ce niveau vital et identitaire. Conséquemment, l'atteinte de cette dimension aux confins requerrait une modalité de réponse sur le plan perceptif, la surface fondamentale.

Dans de tels cas de figure, De M'Uzan (p. 234) circonscrit l'enjeu d'offrir au patient matière à construire une "dalle identitaire», c'est-à-dire une base à partir de laquelle il pourra rebondir depuis un état démentalisé, vers l'aperception de l'intériorité, du monde pulsionnel. Il présente alors l'exemple d'une patiente qui, au sortir d'une séance, embrasse subitement sa 
thérapeute. Suggérant de mettre en suspens la force vertement affectueuse, voire érotique de l'élan, il imagine plutôt lui renvoyer: «Devant la porte, toutes deux, nous nous trouvions debout». Ailleurs, dans la situation qui nous occupe, qu'en est-il de la possibilité, pour une pratique artistique, de répondre à la décharge et à la détresse humaine?

Manifestement, dans ce qu'elle représente, l'œuvre Home-made, Homegrown met, elle aussi, l'excès en suspens, celui de la barbarie marquant le contexte avec lequel des drapeaux sont diffusés. Patient ouvrage depuis un silence pensif, elle n'élance pas la haine ni ne siffle l'amour. L'œuvre reprend chaque image virtuelle en la réduisant à un objet unique qui se voit et qui se touche, aisément, agréablement. Par son retour vers le tangible, par le rétablissement d'un corps tactile qui se laisse être piqué, devient-elle également engagée dans la construction d'une dalle identitaire?

S’impose alors à moi l'image d'un drap-peau.

Si je feins m'extraire de mon propre rapport à l'œuvre, insistant davantage sur la symétrie étrangement inquiétante qu'elle établit avec la réalité sociale, avec la violence du monde, je me dis que l'œuvre tisse une réponse à la précarité des frontières, à la répétition de leur saccage lorsque la quantité prédomine, que des messages sont intromis, que des êtres humains combattent à mort, que des territoires sont envahis. J'observe l'œuvre comme si elle représentait un drap-peau, soit la réfection d'un double, d'une doublure, touché(e)-touchant(e), qui signalerait la survie dans un monde de fous, fous d'anéantissement.

Si je me rappelle que la violence du monde est plus ou moins autrement violence d'un monde mien, le drap-peau que l'œuvre présente ressort à un phénomène intensément vécu. Il devient cette base intermédiaire entre le dedans et le dehors, entre l'étranger et le familier, que ces drapeaux participent à implanter au sein de mon esprit. Entortillés dans ce drap, ou greffés au travers de cette peau: des corps étrangers, des corps de plusieurs violences, corps vacillants, condamnés, sans conscience de leur blessure, des parties de corps sans corps, d'une espèce automatique. Depuis cette trame singulière, me regardent alors des messages qui inscrivent l'inconscience du traumatique parmi des mots réflexifs qui me font témoin, qui me procurent la sensation de voir, d'être touché.

Mouvement de la pensée, avais-je annoncé plus tôt. À la fin de ce parcours, je revisite les origines de ma rêverie pour souligner la modalité perceptive, consolidée par l'œuvre. Citant Didi-Huberman qui invite à «ouvrir les yeux pour éprouver ce que nous ne voyons pas», j'ai peut-être insisté 
davantage sur l'épreuve d'un au-delà du visible, plutôt que sur l'ouverture de mes yeux. Le philosophe évoque une perte dans le visible, et j'ai peut-être insisté davantage sur la perte, au détriment du visible. Or, «devant», j’ai vu des drapeaux, pour ensuite m'inquiéter, jusqu'à errer entre moi et l'autre. J'ai vu des formes taillées dans le textile, pour ensuite voir, et ne plus voir, qu'un mur.

Et si, avant de perdre, et si, pour perdre, l'œuvre donnait à percevoir. Ou plutôt, si l'œuvre donnait à sentir, à incarner les perceptions, à se représenter dans l'espace, soi, en train de percevoir. Et si, pour arriver à rêver dans notre maison, une maison apte à soutenir les échanges avec un dehors qui est aussi le non-lieu du traumatique, du bruit et de l'excès, nous demeurions dépendants du monde, du voisin et de son ouvre d'abord ancrée dans le perceptible. Euvre qui est donnée à sentir, avant même de penser, par-dessous l'abstraction opérée par la pensée. Or, le monde, ou une partie du monde, le voisin, n'est-ce pas cela que nous représentons pour autrui? Celui ou celle-là pour qui nous sommes celui ou celle-là « devant».

Selon Barthes, le discours amoureux est «aujourd'hui d'une extrême solitude» (1977, p.6). Infréquenté, abandonné, délaissé. S’il suppose l'épreuve d'une intériorité, est-ce aussi le territoire intérieur qui s'avère inhabitable, encombré par la quantité? Pour aimer, jusqu'à l'incarnation dans un discours, il y a besoin d'une base. Or, en attendant la base, patiemment, en la recomposant, dalle après dalle, la mise en suspens reste peut-être une façon paradoxale d'aiguiller vers le chant de l'amour. Vers ses traumatismes, sa souffrance.

Pour une base et la possibilité d'inscrire l'excès, de le réécrire, faisons maison. Celleci comprendra-t-elle un dehors œuvrant à la défaire? Complexifiera-t-elle l'attraction réductrice pour des murs étanches, qui cloisonnent le mal au sein de groupes ou de personnes identifiables? S'ouvrirat-elle à l'étrangeté du bruit, provenant d'on ne sait où mais venant, revenant? Aux limites de l'humain, et non sans aide, nous serions capables d'une «inquiétude permanente», selon la formule énoncée par De M'Uzan (2015). Assumerons-nous alors l'incertitude au sein de l'être? Le dérangement, mais celui de toutes ces pensées qui nous convainquent que la violence et sa blessure se trouvent dehors dehors, chez un terroriste, un ennemi ou simplement un autre? Autant de questions arrimées à une langue de l'intelligible.

Le traumatique, quant à lui, rassemble autrement ses mots, soulevant l'interrogation: being with taliban, at home? 


\section{Anthony Bourgeault anthonybourgeault@hotmail.ca}

\section{Note}

1. Je renvoie le lecteur au glossaire que Gagnebin (2015) élabore sur l'assemblage théorique conçu par De M’Uzan.

\section{Références}

Arendt, H. (1971). Considérations morales. Paris : Payot. 1996.

Bachelard, G. (1957). La poétique de l'espace. Paris: Puf. 2001.

Barthes, R. (1977). Fragments d'un discours amoureux. Paris: Seuil.

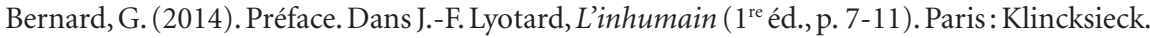
Bersani, L. (1987). Le rectum est-il une tombe? Paris: L'unebévue. 1998.

Bersani, L. (2008). Shame on you. Dans L. Bersani et A. Phillips, Intimacies ( $1^{\text {re }}$ éd., p. 58-97). Chicago: University Press of Chicago. 2008. Format iBook.

Collyer, M. (2015). How Do You Surrender a Drone? Toronto: York University.

Collyer, M. (2006). The series Home-made, Home-grown. Repéré à http://www.milescollyer. com/Miles_Collyer_Home-made_Home-Grown.html

Collyer, M. (2007). Home-made, Home-grown Toronto, Canada. Repéré à http://www.milescollyer.com/Miles_Collyer_Home-made_Home-grown_Toronto. html

Collyer, M. (2008). Home-made, Home-grown, Victoria, Canada. Repéré à http://www. milescollyer.com/Miles_Collyer_Home-made_Home-grown_Victoria.html

Collyer, M. (2010). Conseil des arts de l'Ontario. Repéré à http://www.milescollyer.com/ Miles_Collyer_G_Gallery.html

Collyer, M. (2010a). Some enchanted evenings. Repéré à http://www.milescollyer.com/Miles_ Collyer_Some_Enchanted_Evenings. html

Corin, E. (2010). Présentation: le heurt des langues Psychanalyse et anthropologie en dialogue. Anthropologie et Sociétés, 34 (3), 9-22.

De M'Uzan, M. (1974). S.j.e.m. De l'art à la mort (2e éd., p. 151-163). Paris: Gallimard.

De M'Uzan, M. (1977). Avant-propos. De l'art à la mort (2 éd., p. vii-x). Paris: Gallimard.

De M’Uzan, M. (1984). Les esclaves de la quantité. La bouche de l'inconscient ( $1^{\mathrm{re}}$ éd., p. 155168). Paris: Gallimard, 1994.

De M'Uzan, M. (2004). Addiction et problématique identitaire: le tonus identitaire de base. Aux confins de l'identité ( $1^{\text {re }}$ éd., p. 132-141). Paris: Gallimard, 2005.

De M'Uzan, M. (2005). Le développement identitaire: accomplissements et achoppements. Aux confins de l'identité (1 ${ }^{\text {re }}$ éd., p. 142-165). Paris: Gallimard, 2005.

De M'Uzan, M. (2015). L’inquiétude permanente. Paris: Gallimard. Format iBook.

Dubois, R.-D. (1986). Being at home with Claude. Montréal: Leméac. 2001.

Didi-Huberman, G. (1992). Ce que nous voyons, ce qui nous regarde. Paris: Minuit.

Fédida, P. (1978). L'absence. Paris: Gallimard.

Freud, S. (1905). Trois essais sur la théorie sexuelle. Paris: Gallimard, 1987.

Freud, S. (1908). Le créateur littéraire et la fantaisie. L'inquiétante étrangeté et autres essais (2 éd., p. 29-46). Paris: Gallimard, 1985.

Freud, S. (1919). L’inquiétante étrangeté. L'inquiétante étrangeté et autres essais ( $2^{\mathrm{e}}$ éd., p. 209264). Paris: Gallimard, 1985.

Freud, S. (1920). Au-delà du principe de plaisir. Essais de psychanalyse (2e éd., p. 47-128). Paris: Payot, 2001. 
Gagnebin, M. (2015). Glossaire des principales notions élaborées par l'auteur. Dans M. De M'Uzan, L'inquiétude permanente ( $1^{\text {re }}$ éd., p. 255-362). Paris: Gallimard. Format iBook.

Hutton, J. (2010). Miles Collyer. G Gallery. Repéré à http://www.jenhutton.com/index.php?/ writing/miles-collyer/.

Heather, R. (2011). Miles Collyer. Dans Canadian Art. Spring 2011. Repéré à http://canadianart.ca/reviews/miles-collyer-g-gallery/.

Laplanche, J. (1987). Nouveaux fondements pour la psychanalyse. Paris: Puf.

Laplanche, J. (1990). Implantation, intromission. Le primat de l'autre en psychanalyse (2 éd., p. 355-358). Paris: Flammarion, 1997.

Laplanche, J. (2003). Le crime sexuel. Sexual (1 $1^{\text {re }}$ é, p. 133-152). Paris: Puf, 2007.

Lévinas, E. (1982). Éthique et infini. Paris: Fayard.

Lyotard, J.-F. (1988). Avant-propos: de l'humain. L'inhumain (1 ${ }^{\text {re }}$ éd., p. 13-18). Paris: Klincksieck, 2014.

Lyotard, J.-F. (1990). La phrase-affect (D’un supplément au différend). Misère de la philoso-

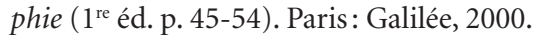

Miller, M. (2010). Betsy Ross and the Making of America. New York: Henry Holt.

Pontalis, J.-B. (1984). Mélancolie du langage. Perdre de vue (1 $1^{\text {re }}$ éd., 193-196) Paris: Gallimard, 1988.

Pontalis, J.-B. (1985). Avertissement de l'éditeur. L'inquiétante étrangeté et autres essais ( $1^{\mathrm{re}}$ éd., p. 7-8). Paris: Gallimard, 1985.

Pontalis, J.-B. (1990). La force d'attraction. Paris: Seuil.

Pontalis, J.-B. (1997). Ce temps qui ne passe pas. Paris: Gallimard.

Quinodoz, D. (2002). Des mots qui touchent: une psychanalyste apprend à parler. Paris: Puf.

Rolland, J.-C. (2006). Avant d'être celui qui parle. Paris: Gallimard.

Roustang, F. (1976). L'étrange familier. Nouvelle Revue de psychanalyse, 14, 85-76.

Scarfone, D. (1994). Ma mère, ce n'est pas elle. De la séduction à la négation. Dans J. Laplanche et coll., Colloque international de psychanalyse (1 $1^{\text {re }}$ é., p. 97-106). Paris: Puf.

Scarfone, D. (2007). Seul ce qui est humain peut nous être étranger. Dans J. André et P. Fédida, Humain/Déshumain (1 ${ }^{\text {re }}$ éd., p. 217-237). Paris: Gallimard, 2007.

Scarfone, D. (2012). Quartiers aux rues sans nom. Paris: Éditions de l'Olivier.

Sécurité publique Canada, (2016). Entités terroristes inscrites, entités inscrites actuellement. Repéré à https://www.securitepublique.gc.ca/cnt/ntnl-scrt/cntr-trrrsm/lstd-ntts/ crrnt-lstd-ntts-fr.aspx 